\title{
USER PREFERRED COLOR COMBINATION DESIGN USING INTERACTIVE GENETIC ALGORITHM
}

\author{
Tad Gonsalves and Ayumi Kawai \\ Department of Information \& Communication Sciences, \\ Sophia University, Tokyo, Japan \\ t-gonsalesophia.ac.jp \\ a2-kawai@nri.co.jp
}

\begin{abstract}
Selecting the right combination of colors in designing a product is often a difficult task. In this paper, the authors propose a decision support system for modeling the color combination design suited for personal preference using interactive Genetic Algorithm (iGA). iGA is different from the traditional GA in that it leaves the evaluation of the objective function to the personal preferences of the user. The iGA interactive system is capable of creating an unlimited number of color combination options taking into consideration the preferences of the user. The user chooses and indicates his/her preferences and directs the process of optimizing the color combination. The final outcome of this user-system interaction is a color-combination design which the user might have not even imagined before he/she began interacting with the system. Users find the system efficient, user-friendly and responding in real-time without causing any user fatigue.
\end{abstract}

\section{KEYWORDS}

Interactive Genetic Algorithm, kansei engineering, optimization, user-preference .

\section{INTRODUCTION}

A lot of design engineering is dedicated to the creation and mixing of colors. Engineering firms devote a lot of their time and resources studying the effects that colors produce on the customers. Kansei engineering, a branch of engineering developed in Japan, concerns itself with the design and manufacture of commercial products taking into consideration the preferences and feelings of the users and customers. Kansei (literally, "emotional / affective engineering") aims at the development or improvement of products and services by translating customer's psychological feelings and needs into product's design domain [4]. Kansei has been used in the design of digital cameras [1], color mapping [2], cars, computers, etc. [4]. Implementing the Kansei is quite a subjective task. Therefore, a decision support system which can model the designs automatically and present a plan of the various designs without heavily biasing the design is necessary. Interactive Genetic Algorithms (iGAs) are optimization techniques used to estimate customers' Kansei [17]. Interactive Genetic Algorithm (iGA) differs from the conventional Genetic Algorithm (GA) in the evaluation of the fitness function. In the conventional GA, the objective function is numerically defined and the algorithm cycles through the stipulated number of generations, while in iGA the evaluation of the objective function is left to the subjective

David C. Wyld et al. (Eds) : CCSIT, SIPP, AISC, PDCTA, NLP - 2014

pp. 169-174, 2014. (C) CS \& IT-CSCP 2014

DOI : $10.5121 /$ csit.2014.4214 
judgment of the user. The termination criterion, too, is in the hands of the user. The aim of GA is to numerically optimize the objective function. In contrast, the aim of iGA is to "optimize" a given design so that it approximates the subjective feelings and judgment of the user. iGA has been used to in designing the layout and lighting of rooms [3], fashions [5], web sites [8], hearing aids [10], and music composition [13], all according to the users' preferences.

This paper deals with the application of iGA in designing color combination of products taking into consideration the user's tastes and preferences. When it comes to the choice of colors or the choice of combination of colors, the user does not normally have a clear idea of the colors. What the user can do is use the color-palette that is available in Microsoft software products and produce a color-combination product of his own choice. However, this would be left entirely to the subjective judgment and creative ability of the user. The result would be a color-combination object designed from a limited number of options - the options being created by the user himself/herself. But in the iGA interactive system, it is the system that creates an unlimited number of options taking into consideration the preferences of the user. The system suggests options based on the preferences indicated by the user. The user then chooses and indicates his preferences and directs the process of optimizing the color combination. The final outcome of this user-system interaction is color-combination design which the user might have not even imagined before he/she began interacting with the system.

This paper is organized as follows: Section 2 describes the interactive Genetic Algorithm. Section 4 presents the iGA system interface along with the internal representation of iGA. Section four presents the various simulation scenarios and results. The paper ends with a short conclusion indicating the direction of future research using iGA.

\section{INTERACTIVE GENETIC ALGORITHM}

The basic difference between GA and iGA can be explained as follows: In the conventional GA, the algorithm automatically cycles through the generations every time producing better fit individuals and stops when the pre-defined number of generations is reached. The user cannot intervene in the evolutionary process from the start to finish. However, in the case of iGA, it is the intervention of the user that drives the evolutionary process.

The application of the iGA is to assist the user in designing the color combination of his/her liking that we propose in this paper consists of the following steps (Figure. 1):

Generation: A population consisting of $\mathrm{N}$ number of individuals is randomly generated. The genes of the individual chromosomes are controlled in such a way that the phenotype colors produced by them are in the range of the initially selected by the user.

Display: The different color combinations and the background produced by the above chromosomes is displayed to the user as shown in Figure. 2 and Figure. 3.

Evaluate: This step is what distinguishes the iGA from the conventional GA. In conventional GA the fitness function is quantified and clearly defined. GA evaluates the solutions based on their fitness function. In iGA program, the evaluation of the fitness of the individual solutions is left to the subjective judgment of the user.

Selection: In the iGA system, there is usually an interface through which the user interacts with the system. The system depicts a feasible number of choices on the interface. From these, the user selects items of his/ her preference usually by using the interface radio buttons and check boxes. 
Crossover: iGA works on the solutions selected by the user and making cross-overs or recombinations to produce new solutions ("offspring") which are closer to the user's preference than the solutions of the previous generation.

Mutation: Some of the newly produced solutions in the above step are subjected to (minor) random mutations. Mutations produce variation in the iGA population and prevent premature convergence of the algorithm.

Termination criterion: This step, too, is very different from the conventional GA. There is no objective pre-defined termination criterion. It rests on the subjective judgment of the user. The user normally terminates the interactive program when he/she is satisfied by the design suggested by the interactive system.

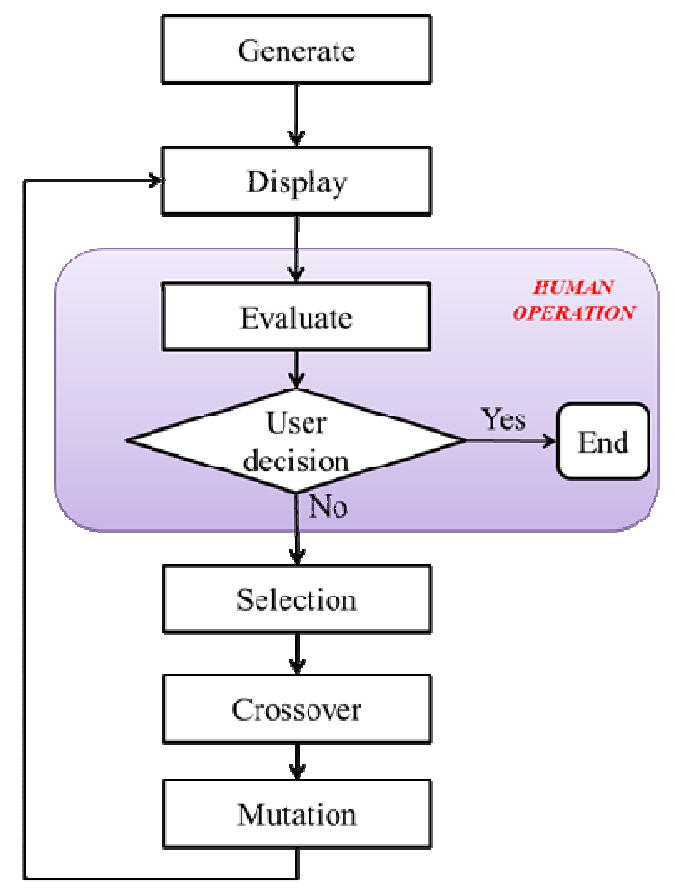

Figure 1. The iGA process

\section{IGA SYSTEM INTERFACE AND IGA}

The interface of the color combination decision support system is shown in Figure. 2 and Figure. 3. It consists of four different objects and a background. The user sets in motion the interactive process by choosing a color for each of the four objects and the background. Several radio buttons are provided on the interface. The user accordingly selects whether the color of a given object and/or the background is to be made lighter or darker or more vivid in the next iGA generation.

The color-combination choices indicated by the user on the interface represent a "solution" in the iGA representation. The aim of the iGA system is to optimize this combination taking into consideration the lighter/darker/vividness of the user selected colors. For this, the system evaluates the fitness of the current solution. It then generates the next solution by incorporating the user's preferences and with a certain amount of randomness. The user further selects the color combination suggested by the system. The user-iGA interaction cycles continue till the user is satisfied by the color combination suggested by the system. If at any time the user is not satisfied 
with the suggestions made by the system, the use can roll-back by pressing the "Back" button. He /she can then proceed from a particular color combination of his/her preference recorded in the history of the interaction.

\section{SIMULATION RESULTS}

The following sub-sections describe two important results: user interaction with the system to produce color combination of his/her liking and the user response about the user-friendliness, efficiency and real-time response of the proposed iGA system.

\subsection{User interaction with iGA system}

One of the convenient uses of the iGA system is to produce tiny variations in the original color combination design indicated by the user. As shown in Figure. 2 the system suggests slight variations in colors, not too far away from the original colors. This procedure can be repeated many times to obtain a desired color combination centered round a theme.

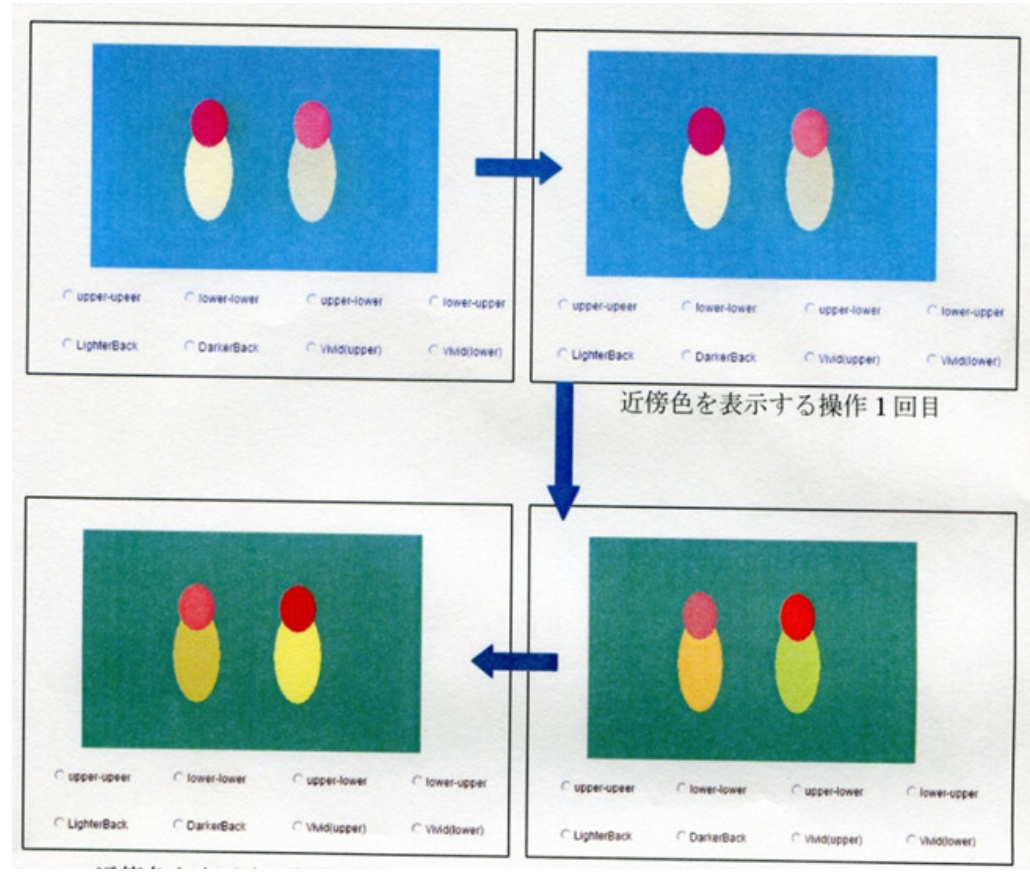

Figure 2. Slight variations in color combination design
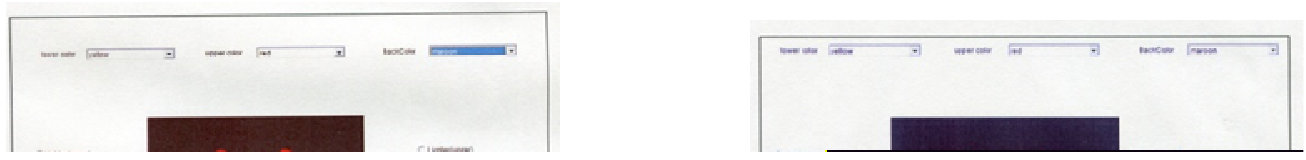

Figure 3. Optimal color combination design produced by the user 
Fig. 4 shows the ideal use of the iGA system. The user chooses different colors for the four distinct objects in the foreground. He/she also chooses the color of the background. In Fig. 4 the user has selected light yellow and red color variations on the foreground objects and maroon color for the background. The user then requests the system to produce lighter objects against a darker background. The final result is obtained after about 5-6 iterations.

\subsection{User feedback}

The author also conducted a small-scale questionnaire with ten volunteers. The results are tabulated in Table 1. On the whole the users find the system easy to use and user friendly. Most of the users could determine a color combination they had in mind within 5-6 cycles of interaction with the system. One of the well-known shortcomings of an interactive system driven by Evolutionary Algorithms is the user fatigue. The user has to interact with the system repeatedly until he/she arrives at an acceptable solution. Several studies have been conducted to measure the user fatigue and ameliorate the situation [11]. In the proposed iGA color combination interactive system all the users' fatigue score was very low. Moreover, they were satisfied with the real-time response of the system.

Table 1: User Questionnaire response

\begin{tabular}{|c|c|c|c|c|}
\hline & Excellent & Good & Fair & Poor \\
\hline $\begin{array}{c}\text { Attainment of preferred color } \\
\text { combination }\end{array}$ & 7 & 3 & 0 & 0 \\
\hline $\begin{array}{c}\text { System accuracy and } \\
\text { efficiency }\end{array}$ & 8 & 2 & 0 & 0 \\
\hline $\begin{array}{c}\text { User-friendliness } \\
\text { Real-time response }\end{array}$ & 8 & 3 & 1 & 0 \\
\hline User-fatigue control & 7 & 2 & 0 & 0 \\
\hline
\end{tabular}

\section{CONCLUSIONS}

In this paper, the author has proposed a decision support system of modeling color combination designs suited for personal preferences by using the interactive Genetic Algorithm (iGA). iGA is an extended version of GA in which the evaluation of the objective function is left to the subjective judgment of the user. The user interacts with the iGA system to design a color combination of his/her preference. The system can produce remarkable color combinations ranging from a slight variation to the original design suggested by the user to highly sophisticated designs that have never been imagined by the user. Simulation experiments show a variety of color combination designs produced by the user interacting with the system. The results of the user questionnaire show that the system is user-friendly, efficient, responds in real-time and does not cause any user-fatigue.

\section{REFERENCES}

[1] Chien-Cheng Chang, Factors influencing visual comfort appreciation of the product form of digital cameras. International jpurnal of Industrial Ergonomics 38 (2008) 1007-1016. 
[2] Y. Fukada, K. Sato, Y. Mitsukura, M. Fukumi, A Design of the Mapping Method of Color and KANSEI, RISP International Workshop on Nonlinear Circuits and Signal Processing (NCSP), pp.7376, China, 2007.

[3] Yosuke Fukada, Keiko Sato, Yasue Mitsukura and Minom Fukumi, The Room Design System of Individual Preference with IGA, International Conference on Control, Automation and Systems,Oct. 2007, Seoul, Korea.

[4] M. Iguchi, Kansei Information Processing, Japan, Ohmsha, 1994.

[5] H. S. Kim and S. B. Cho, "Application of interactive genetic algorithm to fashion design," Engineering Applications of Artificial Intelligence, vol. 13, no. 6, pp. 635 - 644, 2000.

[6] Chih-Chin Lai, andYing-Chuan Chen, A User-Oriented Image Retrieval System Based on Interactive Genetic Algorithm, IEEE Transactions on Instrumentation and measurement, vol.60, No. 10, pp. 3318-3325.

[7] Ali. M. Malkawi, Ravi S. Srinivasan, Yun K. Yi, Ruchi Choudhary, "Decision Support and Design Evolution: Integrating Genetic Algorithms, CFD and Visualization," Automation in Construction, pp.33-44, 2005.

[8] A. Oliver, N. Monmarche, G. Venturini, Interactive Design of Web Sites with a Genetic Algorithm, Proceedings of the IADIS International Conference WWW/Internet, pp.355-362, Lisbon, Portugal.

[9] K. Sato, Y. Mitsukura, M. Fukumi, "Designing of the Color KANSEI Information Map Considering the Individual Characteristic by Using Multivariable Analysis," SICE-ICASE International Joint Confer ence, FP23-4, pp.3706-3710, Busan, Korea, 2006.

[10] H. Takagi, "Application of interactive evolutionary computation to optimal tuning of digital hearing aids," in Int'l Conf. on Soft Computing, IIZUKA'98, World Scientic, 1998.

[11] Takagi, H. ; Pallez, D., Paired comparison-based Interactive Differential Evolution, World Congress on Nature \& Biologically Inspired Computing, 2009. NaBIC 2009.

[12] Sun Yan, Wang Wanliang and Liu Xiaojian, An improved evaluation method for interactive genetic algorithms and its application in product design, 2010 IEEE Fifth International Conference on BioInspired Computing: Theories and Applications (BIC-TA).

[13] N. Tokui, H. Iba, Music Composition with Interactive Evolutionary Computation, in Proc. Generative Art 2000, International Conference on generative Art, Milan, Italy, 2000. 